\title{
BMJ Open Impact of multiple liver resections prior to salvage liver transplantation on survival in patients with recurrent HCC
}

\author{
Zhenhua Hu, ${ }^{1,2,3}$ Qijun Zhang, ${ }^{1,2,3}$ Jie Zhou, ${ }^{1,2,3}$ Zhiwei Li, ${ }^{1,2,3}$ Jie Xiang, ${ }^{1,2,3}$ \\ Lin Zhou, ${ }^{1,2,3}$ Jian Wu, ${ }^{1,2,3}$ Min Zhang, ${ }^{1,2,3}$ Shusen Zheng ${ }^{1,2,3}$
}

To cite: Hu Z, Zhang Q, Zhou J, et al. Impact of multiple liver resections prior to salvage liver

transplantation on survival in patients with recurrent HCC. BMJ Open 2015;5:e008429. doi:10.1136/bmjopen-2015008429

- Prepublication history for this paper is available online. To view these files please visit the journal online (http://dx.doi.org/10.1136/ bmjopen-2015-008429).

ZH and QZ contributed equally.

Received 15 April 2015 Revised 17 July 2015 Accepted 4 August 2015 CrossMark

\footnotetext{
${ }^{1}$ Division of Hepatobiliary and Pancreatic Surgery, Department of Surgery, First Affiliated Hospital, School of Medicine, Zhejiang University, Hangzhou, Zhejiang, China

${ }^{2}$ Key Laboratory of Combined Multi-Organ Transplantation, Ministry of Public Health, Hangzhou, Zhejiang, China ${ }^{3}$ Key Laboratory of Organ Transplantation, Hangzhou, Zhejiang, China
}

Correspondence to Professor Shusen Zheng; shusenzheng@zju.edu.cn

\section{ABSTRACT}

Objectives: Salvage liver transplantation (SLT) is a controversial technique that has been reported to be acceptable for the management of patients with recurrent hepatocellular carcinoma $(\mathrm{HCC})$ after primary hepatic resection (HR). However, whether the number of times liver resection is performed has an impact on survival after SLT has not yet been reported.

Design: Retrospective study.

Setting: The level of care is primary and the study was carried out at only 1 centre.

Participants: The study included 59 patients who underwent SLT for HCC from September 2001 to December 2012. 51 patients underwent HR only once before SLT, while the remaining 8 patients underwent HR more than once before SLT (HR=2 [7], HR=3, [1]).

Primary and secondary outcome measures: In this study, the 1-year, 3-year and 5-year overall and tumour-free survival outcomes between the 2 groups were compared.

Results: There were no significant differences between patients who underwent HR once and those who underwent HR more than once with respect to overall or tumour-free survival after receiving SLT. The 1-year, 3-year and 5-year overall survival rates for patients who underwent HR once were $72.9 \%, 35.3 \%$ and $35.5 \%$ vs $50 \%, 50 \%$ and $50 \%$, respectively ( $p=0.986)$, while the 1-year, 3-year and 5-year tumourfree survival rates for those who underwent $\mathrm{HR}$ more than once were $66.3 \%, 55.3 \%$ and $44.4 \%$ vs $40 \%$, $40 \%$ and $40 \%$, respectively $(p=0.790)$.

Conclusions: There was no significant difference in the survival rate of patients who underwent HR once before SLT and those who underwent HR more than once. This suggests that SLT is a reasonable choice for patients who suffer from recurrent HCC after HR.

Trial registration number: This is a retrospective study and no registry or number is required.

\section{INTRODUCTION}

Hepatocellular carcinoma (HCC) is a disease with increasing worldwide morbidity ${ }^{1}$ as a consequence of the prolongation of human life. Increasing attention is being devoted to

\section{Strengths and limitations of this study}

- This was a retrospective study and proposed the idea of whether the number of hepatic resections (HRs) performed prior to salvage liver transplantation (SLT) has any impact on overall or tumour-free survival.

- This study was retrospective, and all data were selected from a single centre, which limited the sample size.

- This study demonstrated that the number of HRs prior to SLT merely influence on the survival outcomes and suggests that SLT is a reasonable choice for patients who suffer from recurrent hepatocellular carcinoma after HR.

improving the quality of life for HCC incidence in this ageing population, and mortality rates continue to rise. ${ }^{2}$ Many clinical studies have focused on the most effective therapeutic regimen for patients who develop HCC. ${ }^{3}$ At present, hepatic resection (HR) and liver transplantation (LT) are two major curative options available for patients with operable, advanced $\mathrm{HCC}^{4}{ }^{5}$ LT is acknowledged as the primary and optimal strategy available for patients with end-stage liver diseases and/or concomitant HCC, ${ }^{6}$ as it removes the tumour in situ as well as the tissue that has cancerous potential. Sapisochin $e t a l^{7}$ reported significantly better long-term actuarial survival in patients who underwent LT compared with those who received HR. The relatively poor outcome associated with $\mathrm{HR}$ was due to the result of a high HCC recurrence rate. However, the shortage of living-donor organs due to the escalating demand resulting from the increased incidence of $\mathrm{HCC}$ is a serious issue. $^{89}$

Under these circumstances, salvage LT (SLT) was proposed by Majno et al, ${ }^{10}$ which is defined as LT after primary HR (PHR), and has been proven an acceptable 
management strategy for patients with developed recurrent HCC. ${ }^{11}{ }^{12}$ Conversely, the effectiveness of SLT remains doubtful for patients with tumours of greater invasive potential and in patients who have had to undergo more than one surgery. Furthermore, immunosuppression after LT is likely to be a significant challenge for patients who have previously undergone PHR. Although the SLT approach remains controversial, it has been shown that the overall survival following SLT does not differ from that of primary LT (PLT). ${ }^{13}$ In addition, SLT relieves the stress of organ shortage and extends patient lifespan. In the People's Republic of China, the shortage of living donors is an extreme problem, and HCC often recurs following PHR before donors have been matched. In this situation, we typically perform a second liver resection (LR) surgery with the intention of prolonging survival until an appropriate donor can be found.

To the best of our knowledge, whether the number of HRs performed prior to SLT has any impact on overall or tumour-free survival has not previously been reported. Thus, the aim of this study is to evaluate the overall and tumour-free survival between patients who underwent HR only once and those who underwent HR more than once prior to SLT. The results of this study may offer insight into appropriate clinical management strategies for these patients.

\section{METHODS}

\section{Patients}

From September 2001 to November 2012, we performed a single-centre retrospective analysis involving 59 liver recipients, using data obtained from the First Affiliated Hospital of Zhejiang University.

The inclusion criteria were: adult ( $>18$ years old); Chinese nationality; and patients with HCC who underwent previous hepatectomy and received SLT because of tumour recurrence.

The exclusion criteria were: patients with HCC who underwent previous hepatectomy and subsequent LT without record of tumour recurrence (due either to liver failure or as de principle or bridge transplantation); recipients with other types of liver cancer (eg, cholangiocarcinoma); and loss to follow-up.

The 59 participants were divided into two groups based on the number of times they underwent HR before they received SLT: (1) recipients who underwent HR only once before SLT (HR1 group; $n=51$ ) and (2) recipients who underwent LR more than once before SLT (HR2 group; $n=8$ ). Within the latter group, seven patients underwent HR twice and one patient underwent HR three times.

The following variables were compared between these two groups: age, gender, recipient blood type, blood type incompatibility, preoperative $\alpha$-fetoprotein (AFP) level, Model for End-Stage Liver Disease (MELD) score, Child-Pugh score, tumour status (including tumour number, diameter of the largest tumour, sum of tumour diameters and tumour-node-metastasis (TNM) staging) and macrovascular invasion. In addition, operative features (cold ischaemia time, warm ischaemia time and intraoperative blood loss) and post-transplant complications were also included.

The 1-year, 3-year and 5-year overall and tumour-free survival rates between the two groups after SLT were compared. Patient survival was further assessed by the Hangzhou criteria, which we have previously demonstrated to be a feasible candidate selection and prognostic approach for LT selection in HCC recipients. ${ }^{14}$ The Hangzhou criteria require that patients meet one of the two following conditions: (1) total tumour diameter less than or equal to $8 \mathrm{~cm}$ and (2) total tumour diameter more than $8 \mathrm{~cm}$ with histopathological grade I or II and preoperative AFP level $\leq 400 \mathrm{ng} / \mathrm{mL}^{.14}{ }^{15}$ Of the 59 patients, 32 recipients met the Hangzhou criteria, with 28 in the HR1 group and 4 in the HR2 group.

\section{Statistical analysis}

Descriptive statistics were expressed as mean (SD) or median (IQR). Recipient characteristics were compared using the Mann-Whitney $U$ test for continuous variables and Fisher's Exact Test or Continuity Correction for binomial variables, where appropriate. Survival rates were assessed using Kaplan-Meier survival curves. Differences were considered statistically significant if the $p<0.05$; all tests were two sided. All analyses were performed using SPSS 16.0 (SPSS Inc, Chicago, Illinois, USA).

\section{RESULTS}

Patient groups and clinicopathological characteristics

This study evaluated a total of 59 patients treated at our centre who received HR for HCC and later underwent SLT for recurrence. The only difference between these patients was the number of times they underwent HR before LT. Among these patients, 51 underwent only one resection (HR1 group, $\mathrm{n}=51$ ), while 8 underwent HR more than once (HR2 group, $n=8$ ). Of the eight patients in the HR2 group, seven had one re-resection while the other patient had two $(\mathrm{HR}=2, \mathrm{n}=7 ; \mathrm{HR}=3$, $\mathrm{n}=1$ ). Since the individual samples were not sufficiently large, we decided to combine all eight patients into one group (HR2).

There were no significant differences in general characters before SLT between HR1 and HR2 groups, such as gender (male/female: $43 / 8$ vs $7 / 1, \mathrm{p}=0.816$ ), mean age $(47.06 \pm 8.54$ vs $48.13 \pm 7.55, \mathrm{p}=0.799)$, transplant year (before 2008/after 2008: $19 / 32$ vs $1 / 7, p=0.330$ ) and blood type $(p=0.166)$. The LT preoperative AFP level is a very important factor in the Hangzhou criteria. In this study, we found no difference in AFP level between the two groups $(p=0.775)$ : the AFP level in the HR1 group varied from 12.8 to $1176.1 \mathrm{ng} / \mathrm{mL}$ with a median of $232.6 \mathrm{ng} / \mathrm{mL}$, while the range in the HR2 group was $32.1-756.2 \mathrm{ng} / \mathrm{mL}$ with a median of $323.7 \mathrm{ng} / \mathrm{mL}$. Similarly, the MELD (median, 10 vs 10.5) and 
Child-Pugh scores (median, 7 vs 7 ) were not different between groups. As for the diameter of the largest tumour (IQR) and number of tumours, both groups were similar $(\mathrm{p}=0.602$ and $\mathrm{p}=0.588$, respectively). Finally, no differences were found in operative characteristics, including cold ischaemia time, warm ischaemia time and intraoperative blood loss. All statistics are shown in table 1 .

\section{Survival analysis of SLT}

In this study, survival analysis, including overall survival and tumour-free survival, after SLT between patients who underwent HR only once (HR1 group) and those who underwent HR more than once (HR2 group), was compared. There was no significant difference between the two groups in terms of overall survival after SLT; the 1-year, 3-year and 5-year overall survival rates of recipients were $72.9 \%, 35.3 \%$ and $35.5 \%$ vs $50 \%, 50 \%$ and $50 \%$, respectively $(\mathrm{p}=0.986$; figure $1 \mathrm{~A})$. With respect to tumour-free survival, while survival in the HR2 group was inferior to the HR1 group, no statistically significant differences were detected between the two groups: the 1-year, 3-year and 5-year rates were $66.3 \%, 55.3 \%$ and $44.4 \%$ vs $40 \%, 40 \%$ and $40 \%$, respectively $(\mathrm{p}=0.790$; figure 1B).

For recipients fulfilling the Hangzhou criteria, the 1-year, 3-year and 5-year overall survival rates of the HR1 group were $88.4 \%, 68.0 \%$ and $68.0 \%$, respectively, similar to those of the HR2 group $(50.0 \%, 50.0 \%$ and
$50.0 \%$, respectively; $\mathrm{p}=0.150$; figure $2 \mathrm{~A})$. The corresponding 1-year, 3-year and 5-year tumour-free survival rates for those meeting the Hangzhou criteria were $69.5 \%, 69.5 \%$ and $57.9 \%$, respectively, in the HR1 group, and the 1-year and 3-year tumour-free survival rates were $100 \%$ and $50.0 \%$ in the HR2 group, respectively ( $p=0.833$, figure $2 B$ ).

\section{Complications of post-SLT}

The complications of post-transplant were, mainly, postoperative infection, biliary complications, intraoperative bleeding, renal failure, vascular complications and acute rejection. Comparison of each complication shows there was no significant difference between HR1 and HR2 groups ( $p$ value $>0.05$ ). All detailed statistics are shown in table 2.

\section{DISCUSSION}

HCC is the most prevalent type of liver cancer. Curative treatments for HCC that have been used widely and proven effective include HR and LT. In addition, other treatment modalities, such as radiofrequency ablation (RFA) and transarterial chemoembolisation (TACE), should also be considered. Nonetheless, LT remains the most effective curative option for patients with HCC and cirrhosis, ${ }^{16}$ because it removes tumours and tissues that can cause other hepatic diseases. However, in certain areas with a high incidence of HCC and low organ donation rates, especially in the People's Republic of

Table 1 Clinical characteristics of patients in the HR1 and HR2 groups before salvage liver transplantation

\begin{tabular}{|c|c|c|c|}
\hline & HR1 group & HR2 group & p Value \\
\hline Gender (male/female) & $43 / 8$ & $7 / 1$ & 0.816 \\
\hline Age (year) & $47.06 \pm 8.54$ & $48.13 \pm 7.55$ & 0.799 \\
\hline Transplant year (before 2008/after 2008) & $19 / 32$ & $1 / 7$ & 0.330 \\
\hline Blood type & & & 0.166 \\
\hline A & 17 & 1 & \\
\hline$A B$ & 6 & 0 & \\
\hline $\mathrm{B}$ & 15 & 2 & \\
\hline $\mathrm{O}$ & 13 & 5 & 0.337 \\
\hline Blood type incompatible & 9 & 0 & 0.602 \\
\hline Preoperative AFP level, median (IQR), ng/mL & $232.6(12.8-1176.1)$ & 323.7 (32.1-756.2) & 0.775 \\
\hline MELD score & $10(7-15)$ & $10.5(7-15.25)$ & 0.911 \\
\hline TNM classification & & & 0.504 \\
\hline 1 & 13 & 2 & \\
\hline II & 11 & 3 & \\
\hline III & 19 & 1 & \\
\hline IV & 8 & 2 & \\
\hline Child-Pugh score & $7(5-9)$ & $7(5.25-9)$ & 0.781 \\
\hline Diameter of largest tumour, median (IQR), cm & $3(2-5)$ & $3(2.13-4.38)$ & 0.602 \\
\hline Number of tumours, median (IQR) & $2(1-3)$ & $2(1-10.5)$ & 0.588 \\
\hline Sum of tumour diameters, median (IQR), cm & $4.75(3.00-7.88)$ & $4(1.2-12.75)$ & 0.873 \\
\hline Macrovascular invasion & 18 & 1 & 0.381 \\
\hline Cold ischaemia time (hours) & $8.8(7-11.18)$ & $11.58(7.48-12.48)$ & 0.163 \\
\hline Warm ischaemia time (minutes) & $4.5(3.5-5.00)$ & $5.00(4.25-5.00)$ & 0.105 \\
\hline Intraoperative blood loss (mL) & $2500(1500-4500)$ & $5500(2500-10000)$ & 0.460 \\
\hline
\end{tabular}



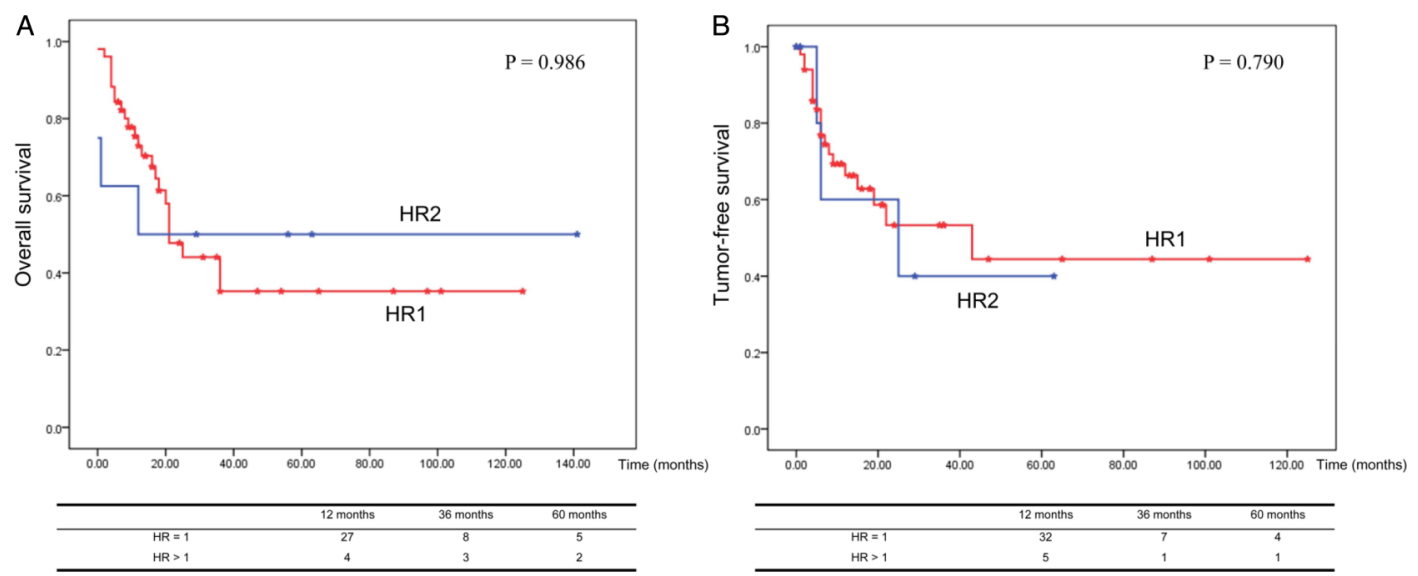

Figure 1 Comparison of overall and tumour-free survival between hepatic resection 1 (HR1) and HR2 groups; (A) overall survival, and (B) tumour-free survival.

China, primary resection is the first-line of treatment for patients with HCC. HR for HCC may be performed with curative intentions following a free resection margin during surgery. ${ }^{17}$ Although this technique may lead to decreased perioperative mortality, the long-term survival is not as good as expected, with a rate of tumour recurrence as high as $70 \%$ within 5 years. ${ }^{4} 18{ }^{19}$ Various factors, such as pathological aspects indicative of tumour invasiveness containing large tumour size, Child-Pugh score and presence of satellite nodules, account for this high recurrence rate. ${ }^{20} 21$

One positive clinical point for patients with HCC recurrence is that they have previously been evaluated for operative re-resection and LT if it was accessible. The evaluation protocol and selection criteria in LT management strategy are similar to those used in the treatment of primary HCC. The Milan criteria were acknowledged worldwide for decades and, recently, the Hangzhou criteria were proven suitable for LT as well. ${ }^{14}$ In the study by $\mathrm{Ho}$ et $a l,{ }^{22} 12.4 \%$ patients with Child-Pugh class A chose re-resection as a treatment option and their 5-year survival was $72 \%$. The remaining patients in the study chose non-surgical methods, including RFA, TACE and support treatment, and no differences were found in the 2-year and 5-year resection rates compared with re-resection. The study by $\mathrm{Ho} e t a l^{22}$ also included nine patients who underwent LT after the first recurrence, which we call SLT, but the authors did not compare the survival rates of this group with the re-resection group.

SLT is a fairly new concept, first presented by Professor Bismuth et $a l^{23}$ in 1999, and has been regarded as the quintessential complementary method of LT and $\mathrm{HR},{ }^{16}{ }^{17}$ as it alleviates the stress of organ donor shortage. ${ }^{24}$ Moreover, SLT extends the time a patient can wait for a donor liver and also provides better life-expectancy over HR.

During recent decades, whether SLT would achieve a curative effect similar to PLT has remained a bone of contention. Therefore, a number of studies have attempted to answer this question. ${ }^{324-27}$ Our previous research ${ }^{11}$ reported that there was no remarkable difference in survival rates between selected recipients of SLT and PLT. Similarly, $\mathrm{Hu}$ et al claimed that, among recipients appropriately selected according to the Hangzhou
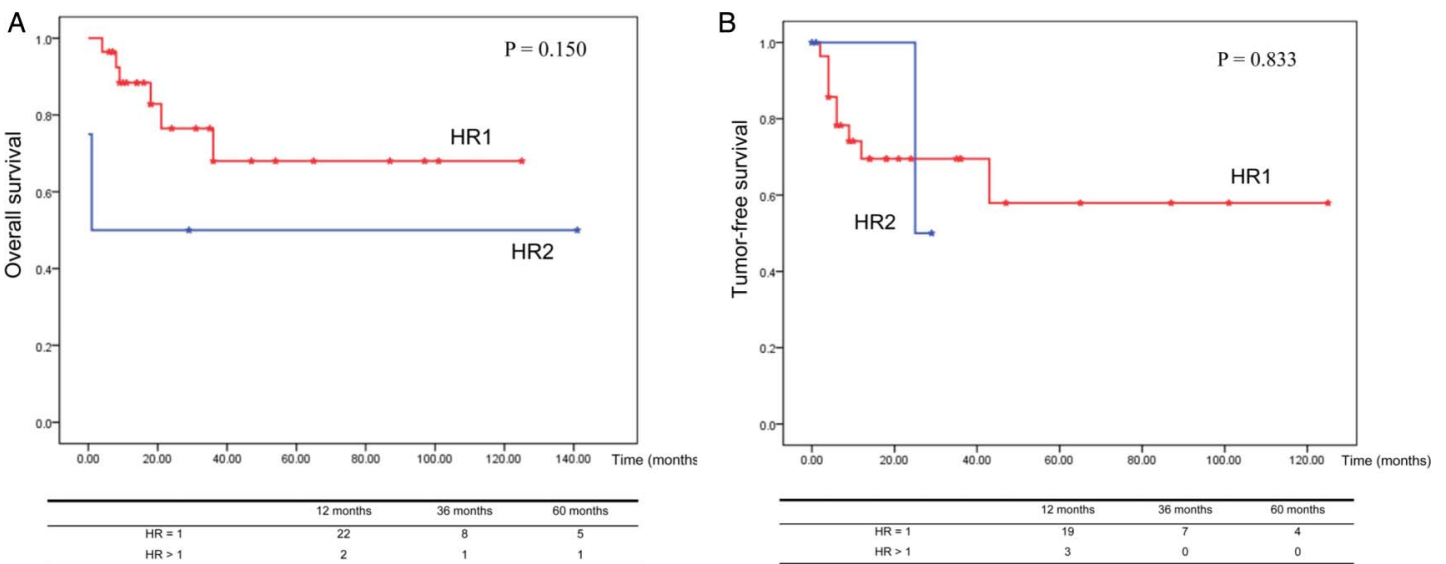

Figure 2 Comparison of overall and tumour-free survival between hepatic resection 1 (HR1) and HR2 groups within the Hangzhou criteria; (A) overall survival, and (B) tumour-free survival. 
Table 2 Comparison of post-transplant complications

\begin{tabular}{|c|c|c|c|}
\hline & HR1 group & HR2 group & p Value \\
\hline \multicolumn{4}{|l|}{ Post-transplant complications } \\
\hline Postoperative infection* & 4 & 0 & 1.0 \\
\hline Biliary complications† & 10 & 0 & 0.386 \\
\hline Intraoperative bleeding & 4 & 2 & 0.183 \\
\hline Renal failure $\ddagger$ & 3 & 0 & 1.0 \\
\hline Vascular complications§ & 7 & 2 & 0.767 \\
\hline Acute rejection & 3 & 1 & 1.0 \\
\hline \multicolumn{4}{|c|}{$\begin{array}{l}\text { *Postoperative infection includes pulmonary infection, catheter-related sepsis, urinary tract infection, wound infection, opportunistic infection. } \\
\text { †Biliary complications include anastomotic biliary strictures, intrahepatic biliary strictures, bile leakage. } \\
\text { fRenal failure includes chronic renal failure, acute renal failure and uraemia (excluding renal failure accompanied by hypertension and } \\
\text { neonatal uraemia). } \\
\text { §Vascular complications include hepatic artery embolism, portal vein embolism, portal vein stenosis/pylethrombosis, hepatic vein/inferior vena } \\
\text { cava stenosis/embolism. } \\
\text { HR, hepatic resection. }\end{array}$} \\
\hline
\end{tabular}

criteria, the 1-year, 3-year and 5-year overall survival rates of SLT recipients were similar to those of PLT recipients: $73.00 \%, 51.77 \%$ and $45.84 \%$ vs $74.49 \%, 55.10 \%$ and $48.81 \%$, respectively $(p=0.260)$. However, the 1-year, 3-year and 5-year disease-free survival rates of SLT recipients were inferior to those of PLT recipients $(p=0.048)$. Prior to our study, Poon and Fan $^{28}$ performed similar research, investigating 107 patients who underwent LT for HCC, and met Mazzafero's criteria on pathological analysis, and concluded that HR prior to transplantation does not increase morbidity or impair long-term survival following LT.

Multiple factors are involved, possibly balancing each other, so that might contribute to an invisible influence on overall and tumour-free survival, factors such as tumour size and number, multifocality, macrovascular invasion, preoperative serum AFP level, histopathological grading, tumour distribution and cirrhosis history. In the present study, the overall and tumour-free survival rates of recipients who underwent HR only once were similar to those who underwent HR more than once prior to SLT. Many factors might be considered to illustrate this result. However, most of the factors aforementioned have been analysed and none of the patients' characteristics that might affect outcome were significantly different between the two groups.

Therefore, we doubt whether liver function status after the first HR would be more important to the evaluation of SLT in the study. Cirrhosis and other background hepatic disorders, such as metabolic disorders and hepatitis, are essential factors that should be taken into consideration during the decision-making process regarding whether it is feasible and whether patients are in a position to undergo surgery. Patients who previously underwent HR before SLT may have poor liver function and their general condition may also be poor; factors that can prevent them from undergoing another HR. In addition, recurrent tumours are not confined to one lobe of the liver; also, the tumour size may be greater than what is suitable for HR. Therefore, SLT may be the only option for patients with recurrent HCC.
Conversely, liver condition and regeneration in patients who accept HR more than once may enable them to undergo a second HR operation. With the presence of certain characteristically favourable histological factors, HR provides an excellent outcome with a low recurrence rate. ${ }^{29}{ }^{30}$ Interestingly, nearly half of patients who undergo $\mathrm{HR}$ have a tumour size less than $2 \mathrm{~cm}$, according to the sizing criteria set forth by Barcelona Clinic Liver Cancer (BCLC) prognosis staging. ${ }^{31}$ The drawback of $\mathrm{HR}$ is that many patients cannot meet the criteria and recurrence is a common phenomenon. However, based on the factors we aforementioned, we doubt whether repeated HRs would dramatically increase either the harm or the benefit of these patients, as the final outcome in patients with multiple $\mathrm{HR}$ is similar to that in patients who undergo only one HR.

We surmise that surgical resection extent is another factor that contributes to the lack of difference in survival between patients with single and multiple HR. For instance, the recurrent HCC tumours may be restricted to one lobe of the liver and lobectomy of a small area is associated with a good prognosis. Thus, even though a patient underwent multiple HRs, it does not necessarily mean that they had more liver area removed than a patient who only underwent one HR. In addition, the interval of HCC recurrence might be an indication of higher invasion of HCC. In brief, all these complicated factors contribute to tumour-free and overall survival rates.

Our study was retrospective, and all data were selected from a single centre, which limited our sample size. In addition, potential confounding factors were adjusted in the single-factor model to decrease the likelihood of the current findings being attributed to selection bias. Therefore, we plan to combine multicentre data in our further studies and analyse other factors in terms of cytobiology and histopathology, if possible.

On the basis of the outcomes of this study, we conclude that SLT is a reasonable approach to use to address the challenge of liver donor shortage and in patients who suffer from recurrent HCC after HR. The number of $\mathrm{HR}$ operations has little influence on 
survival; therefore, treating recurrent HCC regardless of prior HR before SLT or to perform SLT directly after comprehensive evaluation of the general condition of the patients and the donor are both acceptable clinical practices.

Acknowledgements The authors gratefully acknowledge the staff of First Affiliated Hospital of Zhejiang University for their help in original data collection and statistical analysis.

Contributors $\mathrm{SZ}$ and ZH were responsible for study concept and design. QZ and JX performed the experiments. QZ, JZ and ZL carried out the analysis and interpretation of data. MZ and JW operated the HR and SLT, LZ was responsible for perioperative management and recording clinical characteristics. QZ drafted the manuscript. ZH and SZ gave final approval for the version published.

Funding This work was supported by grants from the National S\&T Major Project (number 2012ZX10002017), the Foundation for Innovative Research Groups of the National Natural Science Foundation of China (grant number 81121002), the National Natural Science Foundation of China (grant number 81200331) and the Public Welfare Technology Application Research Project of Zhejiang Province (grant number 2014C33267).

Competing interests None declared.

Patient consent Obtained.

Ethics approval The Committee of Ethics in Biomedical Research of Zhejiang University, China.

Provenance and peer review Not commissioned; externally peer reviewed.

Data sharing statement No additional data are available.

Open Access This is an Open Access article distributed in accordance with the Creative Commons Attribution Non Commercial (CC BY-NC 4.0) license, which permits others to distribute, remix, adapt, build upon this work noncommercially, and license their derivative works on different terms, provided the original work is properly cited and the use is non-commercial. See: http:// creativecommons.org/licenses/by-nc/4.0/

\section{REFERENCES}

1. Yang JD, Roberts LR. Epidemiology and management of hepatocellular carcinoma. Infect Dis Clin North Am 2010;24:899-919, viii.

2. Altekruse SF, McGlynn KA, Reichman ME. Hepatocellular carcinoma incidence, mortality, and survival trends in the United States from 1975 to 2005. J Clin Oncol 2009;27:1485-91.

3. Facciuto ME, Koneru B, Rocca JP, et al. Surgical treatment of hepatocellular carcinoma beyond Milan criteria. Results of liver resection, salvage transplantation, and primary liver transplantation. Ann Surg Oncol 2008;15:1383-91.

4. Jarnagin WR. Management of small hepatocellular carcinoma: a review of transplantation, resection, and ablation. Ann Surg Oncol 2010;17:1226-33.

5. Rahbari NN, Mehrabi A, Mollberg NM, et al. Hepatocellular carcinoma: current management and perspectives for the future. Ann Surg 2011;253:453-69.

6. Wang ZX, Song SH, Teng F, et al. A single-center retrospective analysis of liver transplantation on 255 patients with hepatocellular carcinoma. Clin Transplant 2010;24:752-7.

7. Sapisochin G, Castells L, Dopazo C, et al. Single HCC in cirrhotic patients: liver resection or liver transplantation? Long-term outcome according to an intention-to-treat basis. Ann Surg Oncol 2013;20:1194-202

8. Forner A, Reig ME, de Lope CR, et al. Current strategy for staging and treatment: the BCLC update and future prospects. Semin Liver Dis 2010;30:61-74.

9. Bruix J, Sherman M. Management of hepatocellular carcinoma: an update. Hepatology 2011;53:1020-2.
10. Majno PE, Sarasin FP, Mentha G, et al. Primary liver resection and salvage transplantation or primary liver transplantation in patients with single, small hepatocellular carcinoma and preserved liver function: an outcome-oriented decision analysis. Hepatology 2000;31:899-906.

11. Hu Z, Zhou J, Xu X, et al. Salvage liver transplantation is a reasonable option for selected patients who have recurrent hepatocellular carcinoma after liver resection. PLOS ONE 2012;7 e36587.

12. Kim BW, Park YK, Kim YB, et al. Salvage liver transplantation for recurrent hepatocellular carcinoma after liver resection: feasibility of the Milan criteria and operative risk. Transplant Proc 2008;40:3558-61.

13. Hu Z, Wang W, Li Z, et al. Recipient outcomes of salvage liver transplantation versus primary liver transplantation: a systematic review and meta-analysis. Liver Transp/ 2012;18: 1316-23.

14. Xu X, Lu D, Ling Q, et al. Liver transplantation for hepatocellular carcinoma beyond the Milan criteria. GUT 2015.

15. Zheng SS, Xu X, Wu J, et al. Liver transplantation for hepatocellular carcinoma: Hangzhou experiences. Transplantation 2008;85:1726-32

16. Mazzaferro V, Regalia E, Doci R, et al. Liver transplantation for the treatment of small hepatocellular carcinomas in patients with cirrhosis. N Engl J Med 1996;334:693-9.

17. Neeff $\mathrm{H}$, Makowiec $\mathrm{F}$, Harder $\mathrm{J}$, et al. [Hepatic resection for hepatocellular carcinoma-results and analysis of the current literature]. Zentralbl Chir 2009;134:127-35.

18. Cucchetti A, Vitale A, Del Gaudio M, et al. Harm and benefits of primary liver resection and salvage transplantation for hepatocellular carcinoma. Am J Transplant 2010;10:619-27.

19. Ercolani G, Grazi GL, Ravaioli M, et al. Liver resection for hepatocellular carcinoma on cirrhosis: univariate and multivariate analysis of risk factors for intrahepatic recurrence. Ann Surg 2003;237:536-43.

20. Dahiya D, Wu TJ, Lee CF, et al. Minor versus major hepatic resection for small hepatocellular carcinoma $(\mathrm{HCC})$ in cirrhotic patients: a 20-year experience. Surgery 2010;147: 676-85.

21. Tung-Ping Poon R, Fan ST, Wong J. Risk factors, prevention, and management of postoperative recurrence after resection of hepatocellular carcinoma. Ann Surg 2000;232:10-24.

22. Ho CM, Lee $\mathrm{PH}$, Shau WY, et al. Survival in patients with recurren hepatocellular carcinoma after primary hepatectomy: comparative effectiveness of treatment modalities. Surgery 2012;151: 700-9

23. Bismuth $\mathrm{H}$, Majno $\mathrm{PE}$, Adam R. Liver transplantation for hepatocellular carcinoma. Semin Liver Dis 1999;19:311-22.

24. Can MF, Hughes CB. Primary liver transplantation vs liver resection followed by transplantation for transplantable hepatocellular carcinoma: liver functional quality and tumor characteristics matter. World J Gastrointest Surg 2013;5:5-8

25. Chan DL, Alzahrani NA, Morris DL, et al. Systematic review of efficacy and outcomes of salvage liver transplantation after primary hepatic resection for hepatocellular carcinoma. J Gastroenterol Hepatol 2014;29:31-41.

26. Guerrini GP, Gerunda GE, Montalti R, et al. Results of salvage liver transplantation. Liver Int 2014;34:e96-104.

27. Tranchart $H$, Chirica $M$, Sepulveda $A$, et al. Long-term outcomes following aggressive management of recurrent hepatocellular carcinoma after upfront liver resection. World $J$ Surg 2012;36:2684-91.

28. Poon RT, Fan ST. Resection prior to liver transplantation for hepatocellular carcinoma: a strategy of optimizing the role of resection and transplantation in cirrhotic patients with preserved liver function. Liver Transpl 2004;10:813-15.

29. Eguchi S, Kanematsu T, Arii S, et al. Recurrence-free survival more than 10years after liver resection for hepatocellular carcinoma. $\mathrm{Br} J$ Surg 2011;98:552-7.

30. Nathan H, Schulick RD, Choti MA, et al. Predictors of survival after resection of early hepatocellular carcinoma. Ann Surg 2009;249:799-805

31. Fuks D, Dokmak S, Paradis V, et al. Benefit of initial resection of hepatocellular carcinoma followed by transplantation in case of recurrence: an intention-to-treat analysis. Hepatology 2012;55:132-40. 


\section{Correction}

Hu Z, Zhang Q, Zhou J, et al. The Impact of Multiple Liver Resections Prior to Salvage Liver Transplantation on Survival in Patients with Recurrent HCC. BMJ Open 2015;5:e008429. The 5-year overall survival rate for patients who underwent HR once was $35.3 \%$, not $35.5 \%$, which is the same as the 3-year survival rate. The abstract should be corrected to read as follows:

There were no significant differences between patients who underwent HR once and those who underwent HR more than once with respect to overall or tumour-free survival after receiving SLT. The 1-year, 3-year and 5-year overall survival rates for patients who underwent HR once and those who underwent HR more than once were $72.9 \%, 35.3 \%$ and $35.3 \%$ vs $50 \%, 50 \%$ and $50 \%$, respectively $(\mathrm{p}=0.986)$, while the 1-year, 3-year and 5-year tumour-free survival rates for these two groups were $66.3 \%, 55.3 \%$ and $44.4 \%$ vs $40 \%, 40 \%$ and $40 \%$, respectively ( $\mathrm{p}=0.790$ ).

BMJ Open 2016;6:e008429. doi:10.1136/bmjopen-2015-008429corr1 